University of Nebraska - Lincoln

DigitalCommons@University of Nebraska - Lincoln

Anthony F. Starace Publications

Research Papers in Physics and Astronomy

$8-2015$

Favorable target positions for intense laser acceleration of electrons in hydrogen-like, highlycharged ions

Liang-Wen Pi

University of Nebraska-Lincoln, lpi@unl.edu

S. X. Hu

University of Rochester, shu@lle.rochester.edu

Anthony F. Starace

University of Nebraska-Lincoln, astarace1@unl.edu

Follow this and additional works at: http://digitalcommons.unl.edu/physicsstarace

Part of the Atomic, Molecular and Optical Physics Commons, Elementary Particles and Fields and String Theory Commons, and the Plasma and Beam Physics Commons

Pi, Liang-Wen; Hu, S. X.; and Starace, Anthony F., "Favorable target positions for intense laser acceleration of electrons in hydrogenlike, highly-charged ions" (2015). Anthony F. Starace Publications. 215.

http://digitalcommons.unl.edu/physicsstarace/215

This Article is brought to you for free and open access by the Research Papers in Physics and Astronomy at DigitalCommons@University of Nebraska Lincoln. It has been accepted for inclusion in Anthony F. Starace Publications by an authorized administrator of DigitalCommons@University of Nebraska - Lincoln. 


\title{
Favorable target positions for intense laser acceleration of electrons in hydrogen-like, highly-charged ions
}

\author{
Liang-Wen $\mathrm{Pi}^{1,2}$ S. X. Hu, ${ }^{3}$ and Anthony F. Starace ${ }^{1,2}$ \\ ${ }^{1}$ Department of Physics and Astronomy, University of Nebraska, Lincoln, Nebraska 68588-0299, USA \\ ${ }^{2}$ Kavli Institute for Theoretical Physics, University of California, Santa Barbara, California 93106-4030, USA \\ ${ }^{3}$ Laboratory for Laser Energetics, University of Rochester, Rochester, New York 14623-1299, USA
}

(Received 31 July 2015; accepted 20 August 2015; published online 11 September 2015)

\begin{abstract}
Classical relativistic Monte Carlo simulations of petawatt laser acceleration of electrons bound initially in hydrogen-like, highly-charged ions show that both the angles and energies of the laseraccelerated electrons depend on the initial ion positions with respect to the laser focus. Electrons bound in ions located after the laser focus generally acquire higher $(\approx \mathrm{GeV})$ energies and are ejected at smaller angles with respect to the laser beam. Our simulations assume a tightly-focused linearly-polarized laser pulse with intensity approaching $10^{22} \mathrm{~W} / \mathrm{cm}^{2}$. Up to fifth order corrections to the paraxial approximation of the laser field in the focal region are taken into account. In addition to the laser intensity, the Rayleigh length in the focal region is shown to play a significant role in maximizing the final energy of the accelerated electrons. Results are presented for both $\mathrm{Ne}^{9+}$ and $\mathrm{Ar}^{17+}$ target ions. (C) 2015 AIP Publishing LLC. [http://dx.doi.org/10.1063/1.4930218]
\end{abstract}

\section{INTRODUCTION}

Recent advances in laser technologies have led to the development of petawatt-class lasers, making possible laser intensities of the order of $10^{22} \mathrm{~W} / \mathrm{cm}^{2}$. 1,2 The electric field strengths for these laser intensities are about three orders of magnitude greater than the Coulomb field experienced by a ground state electron in atomic hydrogen. These advances open the regime of highly relativistic light-matter interactions, raising interest in both experimental and theoretical studies of the new phenomena that become possible in this regime. One of these novel phenomena is the acceleration of electrons to $\mathrm{GeV}$ energies. Laser-driven plasma-based electron acceleration is one of the promising methods for obtaining high energy electron beams of good quality. ${ }^{3-7}$ Recently, multi-GeV electron beams have been produced using laser wakefield $^{8,9}$ and plasma waveguide ${ }^{10,11}$ accelerators. Vacuum acceleration is another means for producing high energy electrons. Free electrons can be injected into the focal region of an intense laser beam, where they experience the strong electromagnetic field and are accelerated to $\mathrm{MeV}$ or even $\mathrm{GeV}$ energies. ${ }^{12-18}$ Different schemes have been developed to transfer more energy to the electrons, as well as to reduce the angular divergence of the electron beams, such as those employing radially-polarized laser fields, ${ }^{19-25}$ chirped pulses, ${ }^{26-33}$ an additional magnetic field, ${ }^{34-36}$ or a linearlypolarized Laguerre-Gaussian ("twisted") laser pulse. ${ }^{37}$ In vacuum acceleration, it has been shown theoretically that the angular divergence of the accelerated electron beam can be narrowed by using either a radially-polarized laser pulse ${ }^{24,25}$ or a linearly-polarized Laguerre-Gaussian laser pulse with orbital angular momentum $l= \pm 2 .{ }^{37}$

$\mathrm{Hu}$ and Starace ${ }^{38,39}$ proposed the use of highly-charged ions interacting with an intense laser field as a new scheme for laser acceleration of electrons in vacuum. The ion species are chosen according to the laser intensity so as to ensure that the innermost bound electrons in highly-charged ions are only ionized when the laser field in the pulse approaches its peak intensity. The ionized electrons are then quickly accelerated to nearly the speed of light, surfing on the laser pulse to $\mathrm{GeV}$ energies. In order to better describe actual experimental conditions, realistic laser focusing effects must be included in the theoretical calculations. The most significant one is the relativistic effect induced by an intense external laser field if the electron's ponderomotive energy $U_{p}=e^{2} E_{0}^{2} /\left(4 m \omega^{2}\right)$ is greater than the electron rest mass energy $m c^{2}$, i.e., if the parameter $q=U_{p} /\left(m c^{2}\right)$ is unity or larger, where $E_{0}$ and $\omega$ are the laser's electric field amplitude and angular frequency, respectively. In the present simulations for an $800 \mathrm{~nm}$ Ti:sapphire laser with peak intensity $I \sim 10^{22} \mathrm{~W} / \mathrm{cm}^{2}, q$ is about 1166 , which corresponds to the highly relativistic regime. In addition, the widely-used paraxial Gaussian beam is strictly applicable only for nonrelativistic or weakly relativistic laser-driven electron acceleration. For tightly-focused intense laser fields, however, higher-order corrections to the paraxial approximation are necessary. ${ }^{40-50}$

In this paper, we consider the interaction of an intense linearly-polarized laser pulse with hydrogen-like highlycharged ions (as in Refs. 38 and 39) with an emphasis on the dependence of the accelerated electron energies and angular distributions on the initial positions of the target ions relative to the laser focus. Two prior studies have investigated the dependence of final electron energies on initial electron positions in the laser focal region for both linearly-polarized ${ }^{51}$ and radially-polarized ${ }^{52}$ laser pulses. In each case the electrons are considered to be initially at rest ("test particle approach") and the laser beam is tightly-focused by a parabolic mirror. (For the case of a linearly-polarized pulse, ${ }^{51}$ the parabolic mirror produces laser fields in the focal region that are very different from those we investigate here.) For our case of hydrogen-like highly-charged ions, we investigate 
fields produced by petawatt-class lasers available today and in the near future, where $1 \mathrm{PW}=10^{15}$ Watts. The fields of such lasers can be focused to a small spot size on the order of a wavelength, thereby achieving high intensity of the order of $10^{22} \mathrm{~W} / \mathrm{cm}^{2}$ in the focal region. The relativistic classical trajectory Monte Carlo (CTMC) method is employed to simulate the intense laser ionization and acceleration of electrons in hydrogenlike highly-charged ions. For each laser power level considered, the beam waist in the focus is varied from one to ten times the laser wavelength in our simulations. Our principal result is the demonstration that the final energies and ejection angles of the ionized electrons depend significantly on the initial positions of their parent ions relative to the laser focus. The findings of this paper are different from those in Refs. 51 and 52, as discussed in Section IV.

This paper is organized as follows. In Sec. II, we describe briefly the relativistic quantum mechanical description of an intense laser field interacting with an electron in a highly-charged ion, noting, in particular, its practical limitations in numerical applications. Then, the relativistic classical approach used in our simulations is outlined, including the dynamical equations, the preparation of the ensemble of initial conditions, and the description of the tightly-focused laser beam. In Sec. III, we present the results of our numerical simulations beyond the paraxial approximation and explain qualitatively the key features of the results of our simulations. Finally, in Sec. IV, we summarize our results and present our conclusions. Throughout this paper, we use SI-based atomic units: $e=m_{e}=\hbar=1 /\left(4 \pi \epsilon_{0}\right)=1$.

\section{RELATIVISTIC SIMULATION METHODS}

Laser acceleration of electrons in highly charged ions can be treated as a two-step process: The first step is the ionization of the deeply bound electrons to the continuum; the second step is the acceleration of the ionized electrons by the intense laser field. In the second step, the acceleration of the electrons away from the ion is so rapid that the nuclear Coulomb field can be ignored without loss of accuracy; i.e., the ionized electrons can be treated as free electrons. In the non-relativistic dipole regime, the motion of an electron in a laser field is usually associated with an oscillation along the polarization direction. When the laser intensity increases to the weakly relativistic regime, non-dipole effects (such as the typical figure eight trajectory) caused by the magnetic components of the laser field become important. In the highly relativistic regime, the electron drifts mainly along the laser propagation direction, and the momentum gain is mainly in that direction. In the following, we first discuss in Sec. II A the difficulty of carrying out a relativistic quantum description of the ionization and subsequent acceleration of electrons in highly-charged ions by an intense laser field. We then focus in Secs. II B-IIE on various aspects of the approach we employ: the relativistic classical trajectory Monte Carlo method.

\section{A. Challenges of a relativistic quantum mechanical description}

The motion of a free electron in a plane wave laser field is governed by the time-dependent Dirac equation or, if the spin of the electron is not considered, by the Klein-Gordon equation. Although both equations admit negative energy solutions, these are not considered in this work because the intensity used in our simulations is far below the Schwinger limit of $10^{29} \mathrm{~W} / \mathrm{cm}^{2}$. For a plane wave electromagnetic field, the Dirac equation can be solved exactly and the solutions are called relativistic Volkov states. Since the Volkov states are a complete plane wave basis of free electron solutions of the Dirac equation, they can be used to represent electron wave packets and study their evolution. ${ }^{53,54}$

For ionization of highly-charged ions interacting with a tightly-focused intense laser field, however, exact analytical solutions for either the Dirac or the Klein-Gordon equation do not exist. One must solve either of those equations numerically. Due to the huge excursion amplitude of an ionized electron, a numerical solution of either the Dirac or the Klein-Gordon equation for the Coulomb potential in threedimensional space is beyond the capabilities of today's computers. Therefore, in order to obtain numerical solutions, certain restrictions and approximations are necessary. For example, Ref. 55 treats the case of a hydrogen-like ion interacting with a linearly-polarized laser in two-dimensions and approximates the Coulomb field of the nucleus by a soft Coulomb potential.

Besides direct numerical solutions of the Dirac and Klein-Gordon equations, theorists have also developed relativistic generalizations ${ }^{56,57}$ of both the semiclassical theory of ionization of atoms and ions in an intense laser field and the strong field approximation (SFA). In Ref. 56, the semiclassical theory of ionization by an intense laser field is treated for the cases of linear, circular, or elliptical polarization. In Ref. 57, a Coulomb-corrected SFA is generalized for the relativistic regime (based on the Dirac equation) for the case of ionization of hydrogen-like systems in an intense laser field, treating the laser field exactly and treating the Coulomb field perturbatively by eikonal-Volkov states. In both cases, the laser field is described as a plane electromagnetic wave. Thus, despite these theoretical advances, the quantum description of ionization of an atom or ion in a tightly-focused intense laser field remains a challenge.

\section{B. Relativistic classical trajectory Monte Carlo method}

While existing numerical approaches for solving the relativistic quantum mechanical equations are restricted to twodimensional approximations and soft Coulomb potentials, ${ }^{55}$ the classical relativistic approach is capable of treating the actual Coulomb potential as well as laser-focusing effects in three dimensions. ${ }^{39}$ Although the classical simulations cannot describe the quantum ionization process, they can describe the laser acceleration of electrons after ionization with quantitative accuracy. For the simple case of free electrons interacting with a plane wave laser field, explicit exact analytic expressions for relativistic electron trajectories are presented in Ref. 58 for arbitrary initial positions and velocities. For a focused laser pulse, approximations and numerical integrations are necessary. The classical trajectory Monte Carlo method not only offers a less computationallydemanding alternative to the numerical integration of either 
the Dirac or the Klein-Gordon equations but also provides physical insight into tight-focusing effects, which can significantly change the energy and angle of ionized electrons.

We consider the interaction of an ultraintense laser pulse with a hydrogen-like, highly-charged ion. The laser pulse is assumed to be linearly-polarized along the $x$-axis and to propagate along the $z$-axis. The relativistic classical dynamics of an electron in both a laser field and a Coulomb field is described by the relativistic dynamical equations

$$
\begin{gathered}
d \mathbf{r} / d t=\mathbf{p} / \gamma, \\
d \mathbf{p} / d t=-\left(\mathbf{E}_{C}+\mathbf{E}_{L}+\mathbf{p} \times \mathbf{B}_{L} / \gamma\right),
\end{gathered}
$$

where $\gamma \equiv \sqrt{1+\mathbf{p}^{2} / c^{2}}$ is the usual relativistic factor; $\mathbf{r}$ and $\mathbf{p}$ are the coordinate and mechanical momentum vectors of the electron, respectively; $\mathbf{E}_{L}$ and $\mathbf{B}_{L}$ are the laser electric and magnetic fields; and $\mathbf{E}_{C}$ is the Coulomb electric field. Equation (1) is a coupled system of six first-order differential equations for the six components of the electron's position and momentum vectors. In the absence of an electromagnetic field, solutions of Eq. (1) are relativistic Kepler orbits, which are not closed owing to perihelion shifts. For a plane-wave laser field, analytic solutions have been given for free electrons (i.e., $\mathbf{E}_{C}=0$ ) with arbitrary initial position and momentum. ${ }^{58}$

In general, when both the electromagnetic and Coulomb fields are present, these equations are integrated numerically using the Runge-Kutta method with self-adaptive steps. In classical mechanics, if some electrons move too close to the nucleus, they will very likely fall into the nucleus owing to the Coulomb singularity at $\mathbf{r}=0$. This situation can cause numerical difficulties, because the step size will approach zero. On the other hand, the electron does not fall into the nucleus in reality owing to quantum mechanics. In practice, very few trajectories collapse and for these few, the calculation is stopped whenever the angular momentum of the electron is smaller than $1 / c$; i.e., we neglect these collapsing trajectories. ${ }^{59}$

In our simulations based upon Eq. (1), effects of radiation reaction ${ }^{1,60,61}$ are not treated since the radiative energy loss of the electrons is negligible for the laser parameters used in our simulations. For a $31 \mathrm{PW}$ laser focused to $10 \mu \mathrm{m}$, the radiative energy loss has been estimated in Ref. 39 using the relativistic Larmor formula for the instantaneous radiated power and then integrating it over time along the electron trajectory. It was found that the radiative energy loss is about $10^{-5}$ times smaller than the total energy gain of the electron. The most powerful laser used in our simulations is a $25 \mathrm{PW}$ laser, which is less powerful than that of the laser simulated in Ref. 39, although the peak intensities of the focused laser field are of the same order of magnitude, i.e., $10^{22} \mathrm{~W} / \mathrm{cm}^{2}$. Thus, we expect radiation reaction effects to be negligible in our simulations. Moreover, the radiation reaction force is much smaller than the Lorentz force if the wavelength of the laser field is much larger than the Compton wavelength $\left(3.9 \times 10^{-4} \mathrm{~nm}\right)$ and the laser intensity is much smaller than the Schwinger limit $\left(4.6 \times 10^{29} \mathrm{~W} / \mathrm{cm}^{2}\right)$, both of which criteria are met in our simulations. We also assume that the gasphase ions are dilute enough $\left(10^{23} \mathrm{~m}^{-3}\right.$ or less $)$ that neither collective motions nor plasma effects (such as refractive defocusing and laser wakefield plasma waves) need to be included. ${ }^{62-64}$ Therefore, the integration of Eq. (1) for each electron trajectory is independent, which is ideal for parallel computing. The initial conditions for each electron trajectory are sampled randomly from the prepared relativistic microcanonical ensemble, which is described next.

\section{Relativistic microcanonical ensemble}

Classical trajectory Monte Carlo methods have been used extensively to study collision and ionization processes of atoms and highly-charged ions. ${ }^{65-70}$ The stationary ground state of a target atom or ion is modeled by a microcanonical ensemble in the phase space of the active electron. The distribution function of the microcanonical ensemble is $\rho(\mathbf{r}, \mathbf{p}) \propto \delta\left(\varepsilon_{g}-\varepsilon(\mathbf{r}, \mathbf{p})\right)$, in which $\varepsilon_{g}$ is the relativistic quantum ground state energy of a hydrogen-like ion and $\varepsilon(\mathbf{r}, \mathbf{p})$ is the relativistic energy of an electron in a Coulomb potential

$$
\varepsilon(\mathbf{r}, \mathbf{p})=c^{2} \sqrt{1+p_{r}^{2} / c^{2}+L^{2} / r^{2} c^{2}}-Z / r,
$$

where $r$ is the radial position of the electron, $p_{r}$ is its radial momentum, and $L$ is its angular momentum. Since the relativistic Kepler problem is analytically solvable, ${ }^{71}$ one obtains the following equation for the electron orbit:

$$
\begin{aligned}
\frac{1}{r}= & \frac{\sqrt{c^{2} L^{2} \varepsilon_{g}^{2}-c^{4}\left(c^{2} L^{2}-Z^{2}\right)}}{c^{2} L^{2}-Z^{2}} \\
& \times \cos \left(\sqrt{1-Z^{2} / c^{2} L^{2}} \phi\right)+\frac{Z \varepsilon_{g}}{c^{2} L^{2}-Z^{2}},
\end{aligned}
$$

where polar coordinates $(r, \phi)$ are used since the angular momentum $L$ is a constant and since the electron's motion in the Coulomb potential is planar.

To prepare this initial ensemble numerically in a computer simulation, each point in phase space needs to be determined from a set of initial parameters. The first parameter, $\varepsilon(\mathbf{r}, \mathbf{p})$, is set equal to the relativistic ground state energy, $\varepsilon_{g}$, of the hydrogen-like ion. The second parameter, the squared angular momentum $L^{2}$, is randomly chosen between $Z^{2} / c^{2}$ and $Z^{2} /\left(c^{2}-\varepsilon_{g}^{2} / c^{2}\right)$, within which range $L^{2}$ is uniformly distributed. ${ }^{59}$ With specified ground state energy $\varepsilon_{g}$ and angular momentum $L$, the relativistic Kepler orbit is determined using Eq. (3), which can be assumed without loss of generality to lie in the $x y$ plane (in Cartesian coordinates). The initial position $\left(r_{0}, \phi_{0}\right)$ of a trajectory can be randomly sampled along the Kepler orbit. ${ }^{59}$ Then, the corresponding radial momentum $p_{r}$ of the electron can be determined from the total energy $\varepsilon(\mathbf{r}, \mathbf{p})$. So far, we have obtained the initial position coordinates and momenta $\left(r_{0}, p_{r}, \phi_{0}, L\right)$, which can be easily converted to Cartesian coordinates $\left(x_{0}, p_{x_{0}}, y_{0}, p_{y_{0}}\right)$ in the $x y$ plane. The last step is to rotate this single phase space point in the $x y$ plane into three dimensions using an arbitrary set of Euler angles. In this way, one obtains a set of initial position and momentum coordinates (representing a single point in phase space) for a single electron trajectory. One then repeats this procedure to prepare a relativistic microcanonical ensemble containing 500000 points in phase space. 
Having prescribed the initial conditions for the active electron, we require next an accurate description of the electromagnetic field in order to integrate Eq. (1).

\section{Nonparaxial Gaussian beams}

When analyzing and modeling experiments, it is important to describe both the temporal profile and the spatial distribution of the laser fields in the focal area correctly, i.e., they must satisfy Maxwell's equations. In many applications, the Gaussian beam description is often used to represent the fields of a focused laser beam propagating along the $z$-axis. When the beam divergence angle is small, i.e., the Gaussian beam waist $w_{0}$ is much greater than the laser wavelength $\lambda$, the beam is generally well-described by the paraxial approximation, which assumes the laser beam is a transverse electromagnetic wave. In order to attain ultra-intense laser intensities for accelerating electrons, however, the laser beam is typically focused to the diffraction limit, which means the beam waist $w_{0}$ is of the order of the laser wavelength $\lambda$. Under such a "tight-focusing" condition, both longitudinal electric and magnetic fields appear as well as other non-paraxial field components in all three dimensions. The laser beam is thus no longer a transverse wave; higher-order corrections to the paraxial approximation must be introduced to ensure that Maxwell's equations are satisfied. Fields of a Gaussian beam beyond the paraxial approximation have been discussed in a number of references. ${ }^{12,40-50}$ Here, we give only a brief overview of the theory we have adopted for our simulations, and mainly follow the work and notations of Ref. 43, but using SI-based atomic units.

Consider a monochromatic beam with frequency $\omega$ and wave number $k=\omega / c$ (where $c \simeq 137$ a.u. is the speed of light in vacuum) that is linearly-polarized along the $x$-axis and propagating along the $z$-axis. Its electric and magnetic fields can be derived from a linearly-polarized vector potential $\mathbf{A}$, which satisfies the wave equation

$$
\nabla^{2} \mathbf{A}-\frac{1}{c^{2}} \frac{\partial^{2} \mathbf{A}}{\partial t^{2}}=0
$$

and a scalar potential $\Phi$, which is related to the vector potential A by the Lorentz condition

$$
\nabla \cdot \mathbf{A}+\frac{1}{c^{2}} \frac{\partial \Phi}{\partial t}=0
$$

We assume the linearly-polarized vector potential has the form

$$
\mathbf{A}=\mathbf{e}_{x} A_{0} \psi(x, y, z) e^{i\left(\omega t-k z+\varphi_{0}\right)}
$$

where $\mathbf{e}_{x}$ is the polarization unit vector along the $x$-axis, $A_{0}$ is a constant amplitude, $\varphi_{0}$ is the initial phase, and $\psi(x, y, z)$ is the spatial profile that must be determined. Inserting (6) into (4) gives the Helmholtz equation,

$$
\nabla^{2} \mathbf{A}+k^{2} \mathbf{A}=0
$$

To facilitate the derivations, we normalize the spatial coordinates relative to the characteristic lengths of the beam focus, i.e., the Gaussian beam waist, $w_{0}$, and the Rayleigh length, $z_{R} \equiv \frac{1}{2} k w_{0}^{2}$, as follows:

$$
\xi=\frac{x}{w_{0}}, \quad \eta=\frac{y}{w_{0}}, \quad \zeta=\frac{z}{2 z_{R}} .
$$

In these scaled coordinates, the Helmholtz equation becomes

$$
\left(\frac{\partial^{2}}{\partial \xi^{2}}+\frac{\partial^{2}}{\partial \eta^{2}}-2 i \frac{\partial}{\partial \zeta}\right) \psi=-s^{2} \frac{\partial^{2} \psi}{\partial \zeta^{2}}
$$

where the diffraction parameter, $s$, is defined as

$$
s \equiv \frac{w_{0}}{2 z_{R}}=\frac{1}{k w_{0}}=\frac{1}{2 \pi} \frac{\lambda}{w_{0}} .
$$

If the Gaussian beam waist $w_{0}$ is assumed larger than the wavelength $\lambda$ (which is the case in our simulations), then the dimensionless parameter $s$ is small and may be used to expand $\psi$ in Eq. (9) perturbatively, as follows:

$$
\psi=\sum_{n=0}^{\infty} s^{2 n} \psi_{2 n} .
$$

Inserting (11) into (9) and equating the coefficients of each power of $s^{2}$ on both sides, one obtains the following equations for the coefficients $\psi_{2 n}$ :

$$
\begin{gathered}
\left(\frac{\partial^{2}}{\partial \xi^{2}}+\frac{\partial^{2}}{\partial \eta^{2}}-2 i \frac{\partial}{\partial \zeta}\right) \psi_{0}=0 \\
\left(\frac{\partial^{2}}{\partial \xi^{2}}+\frac{\partial^{2}}{\partial \eta^{2}}-2 i \frac{\partial}{\partial \zeta}\right) \psi_{2 n}=-\frac{\partial^{2} \psi_{2 n-2}}{\partial \zeta^{2}} \quad(n \geq 1) .
\end{gathered}
$$

Equation (12a) gives the familiar paraxial approximation for the fields. Solutions of the two-term recursion relation (12b) have been obtained for $n=1$ and 2, corresponding to the following 2nd-order and 4th-order corrections: ${ }^{42,43}$

$$
\begin{gathered}
\psi_{0}=i Q \exp \left(-i \rho^{2} Q\right), \\
\psi_{2}=\left(2 i Q+i \rho^{4} Q^{3}\right) \psi_{0}, \\
\psi_{4}=\left(-6 Q^{2}-3 \rho^{4} Q^{4}-2 i \rho^{6} Q^{5}-0.5 \rho^{8} Q^{6}\right) \psi_{0},
\end{gathered}
$$

where

$$
\rho=\xi^{2}+\eta^{2}, \quad \text { and } \quad Q=\frac{1}{i+2 \zeta} .
$$

Substituting the results in (13) into Eqs (11) and (6), the vector potential takes the form

$$
\mathbf{A} \approx \mathbf{e}_{x} A_{0}\left(\psi_{0}+s^{2} \psi_{2}+s^{4} \psi_{4}\right) e^{i\left(\omega t-k z+\varphi_{0}\right)},
$$

which satisfies the Helmholtz equation up to fifth-order in $s$. Expressing the scalar potential in terms of the vector potential (15) using Eq. (5), the electric and magnetic fields are obtained from the vector potential as follows: 


$$
\mathbf{E}=-\left(i c^{2} / \omega\right) \nabla(\nabla \cdot \mathbf{A})-i \omega \mathbf{A}, \mathbf{B}=\nabla \times \mathbf{A} .
$$

The electromagnetic fields obtained by this procedure were given in Ref. 49 up to the eleventh-order correction $(n=5)$. The fields in Ref. 49 are not symmetric, since the vector potential $\mathbf{A}$ is assumed parallel to the $x$-axis, leading to $B_{x}=0$ at all times. In order to obtain a more symmetrical set of equations for higher order field components, in Ref. 43 , the derivation was repeated assuming that the vector potential $\mathbf{A}$ is polarized along the $y$ axis, and then the electric and magnetic fields derived from the two procedures were averaged (A similar symmetrization procedure was used in Ref. 12.) Except for an overall phase factor $-i$, the unsymmetrized electromagnetic fields in Ref. 49 and the symmetrized fields in Ref. 43 are the same up to order $s^{1}$

$$
\begin{gathered}
E_{x}^{(0)}=E_{0} \psi_{0} e^{i \varphi}, \\
B_{y}^{(0)}=\frac{E_{0}}{c} \psi_{0} e^{i \varphi}, \\
E_{z}^{(1)}=-2 s Q \xi E_{0} \psi_{0} e^{i \varphi}, \\
B_{z}^{(1)}=-2 s Q \eta \frac{E_{0}}{c} \psi_{0} e^{i \varphi} .
\end{gathered}
$$

In Eq. (17), the transverse components $E_{x}^{(0)}$ and $B_{y}^{(0)}$ are the paraxial fields, and the longitudinal components $E_{z}^{(1)}$ and $B_{z}^{(1)}$ are the lowest-order non-paraxial fields, where $\varphi \equiv \omega t$ $-k z+\varphi_{0}$ is the phase and $E_{0} \equiv \omega A_{0}$ is the amplitude of the electric field at the center of the laser beam focus (i.e., at $x=y=z=0$ ). For more details on higher order corrections, please see Refs. 43 and 49.

Although the two sets of formulas for the non-paraxial fields in Refs. 43 and 49 differ for terms of order $s^{2}$ and higher, for the largest value of the parameter $s$ employed in the present work (i.e., $s=0.127$, corresponding to a beam waist $w_{0}=1 \mu \mathrm{m}$ ), both sets of formulas give essentially identical numerical results for the ionized electron trajectories. Specifically, for both individual ionized electron trajectories and the statistical distribution of final electron energies and angles, we employed the unsymmetrized formulas of Ref. 49 up to eleventh-order and compared the calculated results with those obtained using the symmetrized formulas of Ref. 43 up to fifth-order. The results of the two calculations were nearly identical, with only minor differences occurring for the uninteresting cases of large scattering angles and small final energies. Based on these test results, we calculated all other cases employing the formulas of Ref. 43 with corrections to the paraxial approximation up to the fifth-order.

Using the real parts of the laser fields in Ref. 43, the power of the laser beam is calculated by integrating the longitudinal component of the Poynting vector over the transverse plane through the focus at $z=0$ and averaging it over time. The resulting average beam power (containing higherorder corrections due to tight-focusing effects) is:

$$
P=\frac{\pi w_{0}^{2}}{2} I_{0}=\frac{\pi w_{0}^{2}}{4} c \varepsilon_{0} E_{0}^{2}\left(1+s^{2}+1.5 s^{4}\right),
$$

where $I_{0}$ is the peak intensity at the center of the laser focus and $\varepsilon_{0}=1 / 4 \pi$ a.u. is the vacuum permittivity.

So far, the electric and magnetic fields have been derived under the assumption that the laser beam is a continuous wave instead of a pulse. For long laser pulses, this assumption should be a good approximation, as has been discussed in Refs. 12 and 72. In this work, we consider a linearly-polarized laser pulse propagating along the $z$-axis, having an electric field envelope described by the Gaussian function

$$
F(t-z / c)=\exp \left[-4 \ln 2 \frac{(t-z / c)^{2}}{\tau^{2}}\right],
$$

where $\tau$ is the duration (i.e., the full width at half maximum) of the electric field of the laser pulse. For the laser beams we consider in Sec. III, $\tau$ is about $10 \times 2 \pi / \omega$, i.e., about 10 cycles. The time $t=0$ is the moment when the peak of the laser pulse reaches the center of the focal region, $z=0$. In our simulations, the electric and magnetic fields actually used are the real parts of the laser fields in Ref. 43 multiplied by the Gaussian envelope (19).

\section{E. Tests for validity of our simulations}

With a properly-prepared microcanonical ensemble and an accurate description of the laser beam, the over-the-barrier ionization rate for hydrogen-like ions can be calculated by determining the percentage of the initial electrons having a total energy exceeding their rest energy $m c^{2}$ at the end of the laser pulse. Typically, one has to calculate at least a few thousand trajectories until the ionization rates and the final electron energy distributions become statistically stable. The ionization fractions for a wide range of hydrogen-like ions, exposed to intense laser fields with intensities ranging from $10^{18} \mathrm{~W} / \mathrm{cm}^{2}$ to $10^{26} \mathrm{~W} / \mathrm{cm}^{2}$, were calculated using relativistic classical Monte Carlo simulations. ${ }^{69}$ In fact, this procedure is useful for determining ultrastrong laser field intensities in experiments, since the measurement of the ionization fraction is a well-established experimental technique and the ionization fraction has a monotonic dependence on laser intensity. In order to check the validity and accuracy of our simulations, we calculated the ionization fractions for hydrogen-like ions with $Z=18,20,25$, and 26 as functions of laser intensity, as shown in Fig. 1. Our results for hydrogen-like ions with $Z=20$ and 25 agree very well with those shown in Fig. 1 of Ref. 69.

\section{RESULTS AND DISCUSSION}

After confirming the validity of our methods, we investigated laser acceleration of electrons in hydrogen-like, highly-charged ions for different laser powers and focus beam waists, with the goal of demonstrating the dependence of the final energy and angular distributions of the accelerated electrons on the initial locations of their parent ions. The laser fields of the pulses in our simulations have a wavelength of $800 \mathrm{~nm}$ and a Gaussian temporal envelope [see Eq. (19)] with FWHM, $\tau$, of $10 T$ (about 27 fs for an $800 \mathrm{~nm}$ laser), where $T=\lambda / c=2 \pi / \omega$ is the laser period. Taking 


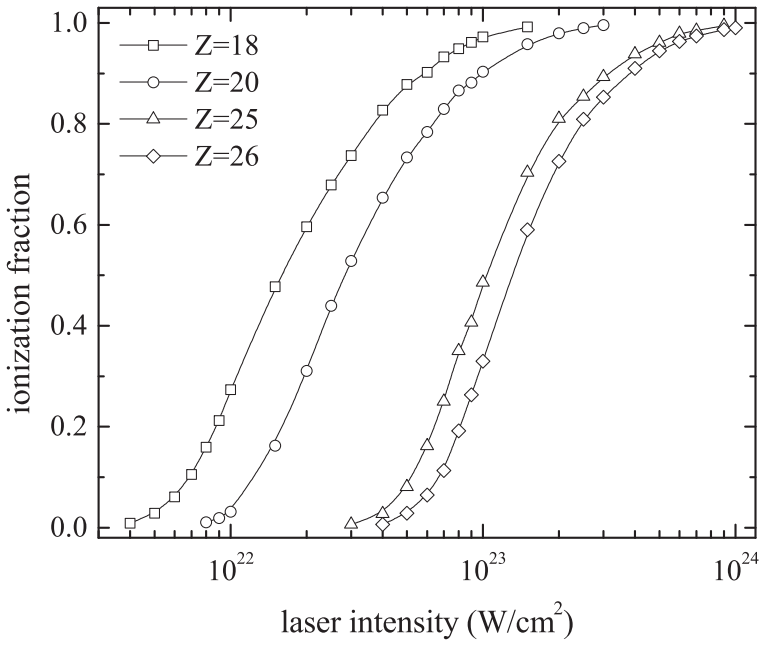

FIG. 1. Over-the-barrier ionization fraction as a function of peak laser intensity at the center of the laser focus for hydrogen-like ions with nuclear charges $Z=18,20,25$, and 26 . The ionization fractions are calculated at the end of a single-cycle Gaussian-shaped laser pulse of wavelength $\lambda=1054 \mathrm{~nm}$ and beam waist $w_{0}=10 \mu \mathrm{m}$. Symbols indicate the calculated results; solid lines are added to guide the eye.

note of the laser powers available today and in the near future, our simulations were carried out for three different average powers of $1 \mathrm{PW}, 5 \mathrm{PW}$, and $25 \mathrm{PW}$. Laser peak intensities ranging from $10^{20} \mathrm{~W} / \mathrm{cm}^{2}$ to $10^{22} \mathrm{~W} / \mathrm{cm}^{2}$ may be achieved by focusing the laser beam to a beam waist from $10 \mu \mathrm{m}$ to $1 \mu \mathrm{m}$. The relation between the average laser power, the peak intensity, and the Gaussian beam waist is given by Eq. (18). The hydrogen-like ions are assumed to be distributed uniformly within a cylindrical volume (having a radius of the Gaussian beam waist, $w_{0}$, and a length twice that of the Rayleigh length, $z_{R}$, oriented along the $\mathrm{z}$ axis and centered about the origin). The distribution of initial electron positions and momenta inside each ion are given by a microcanonical ensemble, mimicking the quantum ground state of a hydrogen-like ion.

Each individual electron trajectory begins to evolve when the laser pulse just reaches the initial location of its parent ion, which is randomly chosen within the cylindrical volume mentioned above. The evolution of each trajectory ends under any of the following three circumstances:

(1) At any moment (before the laser pulse passes the ion location) at which the angular momentum of the electron is smaller than $1 / c$, in which case the electron is considered as falling into the nucleus. ${ }^{59}$

(2) When the laser pulse has passed the ion location and the total energy of the electron is still less than its rest mass energy, in which case the electron no longer has any possibility to be ionized.

(3) At any moment (before the laser pulse passes the ion location) that the total energy of the electron is greater than its rest mass energy, in which case the electron is considered to have been ionized. Its trajectory is then computed until it falls behind the laser pulse and its momentum becomes constant. The time (in the lab frame) for this to occur is typically of the order of ten thousand laser cycles.
The above simulation criteria are repeated until at least a few thousand ionized electron trajectories have been obtained in order that the energy and angular distributions are converged.

\section{A. Timing of ionization}

Most literature in the field of vacuum acceleration deals with the scheme of injecting free electrons into the focus of a laser beam. These electrons will be reflected from, transmitted through, or "captured" by the laser beam, but only those captured can experience higher intensity and thereby have a good chance of being accelerated to higher energy. ${ }^{14,15}$ The idea of using highly charged ions ${ }^{38,39}$ is to keep the electrons bound through the turn-on stage of the laser pulse so that the electrons become ionized near the peak of the laser pulse, thus experiencing the highest laser intensity. Reflection and transmission in this scheme are no longer a problem.

We illustrate this idea in Fig. 2 by recording the number of electrons ionized in each laser cycle for hydrogenlike $\mathrm{Ar}^{17+}$ ions interacting with a $1 \mathrm{PW}, 800 \mathrm{~nm}$ laser beam focused to $2 \mu \mathrm{m}$. The laser fields in our simulations include up to fifth-order corrections in the focal region, given in Ref. 43 , in which the initial phase $\varphi_{0}$ is set to zero in all our simulations, since the laser pulse is fairly long and hence the initial phase is not important. One can see in Fig. 2 that ionization occurs most probably in the 12th cycle, whereas the peak intensity occurs in the 13th cycle. Electrons ionized during the 9 th to 16 th cycles account for $91 \%$ of the ionization events.

Figure 2 also indicates how to choose a suitable target ion according to the laser parameters. If one chooses an ion with nuclear charge smaller than 18 , most electrons are ionized long before the laser peak arrives. On the other hand, if one chooses an ion with nuclear charge larger than 18 , most ionization will occur near the peak intensity, but the overall ionization rate may be too low to be efficient. The most

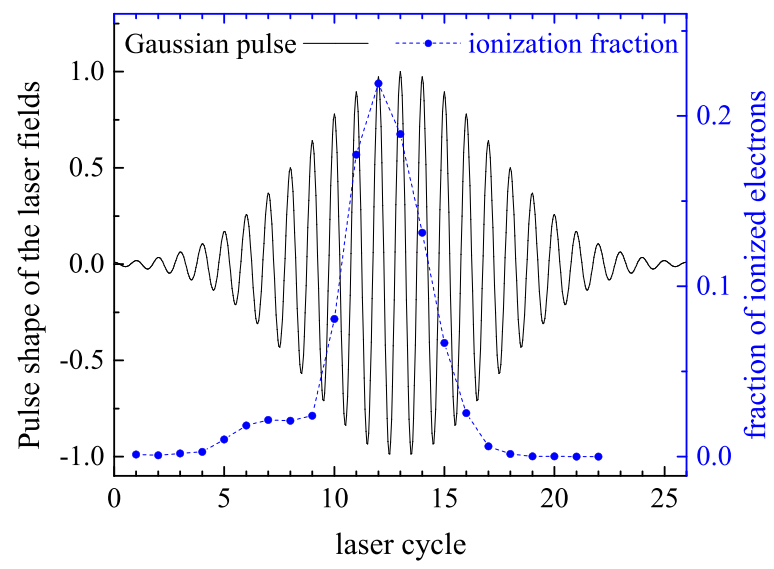

FIG. 2. Electron ionization as a function of laser cycle within the laser pulse envelope. Solid line (black): Real part of the temporal dependence of the laser fields (17), i.e., $\cos \left(\omega t-k z+\varphi_{0}\right)$ multiplied by the Gaussian envelope (19). The initial phase $\varphi_{0}$ is zero and the FWHM, $\tau$, is 10 laser cycles. Circles connected by the dashed line (blue): Each circle represents the fraction of ionized electrons during one laser cycle for hydrogen-like $\mathrm{Ar}^{17+}$ ions interacting with a $1 \mathrm{PW}, 800 \mathrm{~nm}$, Gaussian-shaped laser beam focused to $2 \mu \mathrm{m}$, resulting in a peak intensity of $1.59 \times 10^{22} \mathrm{~W} / \mathrm{cm}^{2}$. 
suitable target ion is best found by trail and error, keeping these points in mind.

\section{B. Final energy and ejection angle distributions}

In this section, we show simulation results for highlycharged ions interacting with an intense laser beam having various intensities and focal beam waists. We find that the final energies and ejection angles of the ionized electrons depend on their initial locations relative to the laser focus. Electrons ionized from ions initially located after the focus $\left(z_{0}>0\right)$ are accelerated to higher energies and ejected at smaller angles than those located before the focus $\left(z_{0}<0\right)$. We also present qualitative explanations for the underlying physics of these findings.

The contour plots in Fig. 3 show the dependence of the distribution of the final electron energies and ejection angles on target ion locations relative to the laser focus for two laser beam waists. Our calculations of these results include electromagnetic field corrections up to fifth-order in the parameter $s$ (cf. Eq. (10)). The ejection angle $\theta$ is defined as $\theta=\arccos \left(p_{z} /|\mathbf{p}|\right)$, where $p_{z}$ and $\mathbf{p}$ are the $z$ component and total momentum at the end of the electron trajectory, respectively. The final kinetic energy of an ionized electron is $\varepsilon=(\gamma-1) m c^{2}$. In their interactions with a $1 \mathrm{PW}$ laser beam focused to a beam waist of $1 \mu \mathrm{m}$, electrons initially located before the focus $\left(z_{0}<0\right)$ are most likely accelerated to $0.05 \mathrm{GeV}$ and ejected at $9^{\circ}$ [Fig. 3(a)], while electrons initially located after the focus $\left(z_{0}>0\right)$ are most likely accelerated to $0.15 \mathrm{GeV}$ and ejected at $6^{\circ}$ [Fig. 3(b)]. A similar

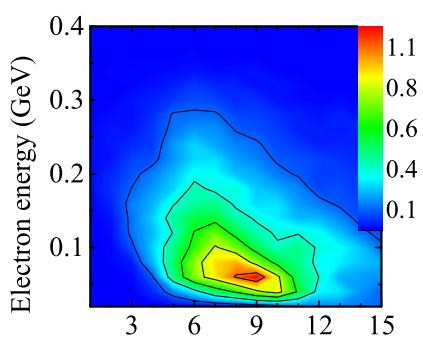

(a)

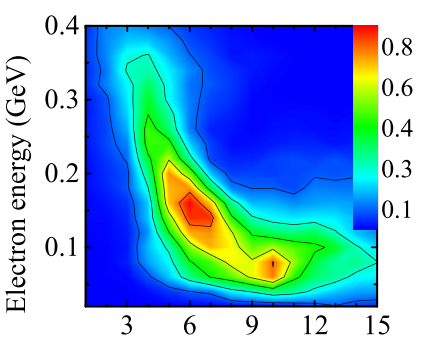

(b) Ejection angle $\theta$ (deg)

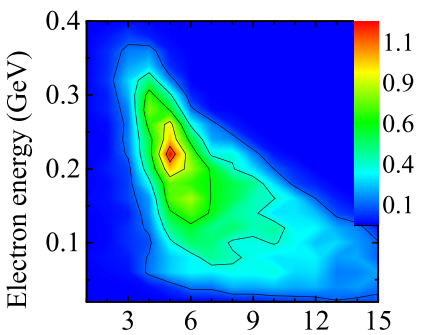

(d) Ejection angle $\theta(\mathrm{deg})$ (c)

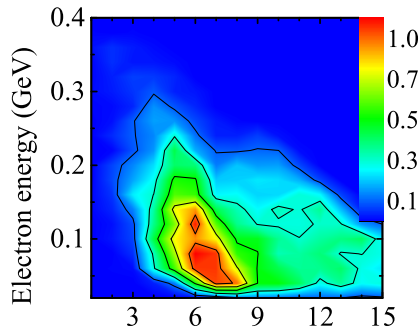

Ejection angle $\theta(\mathrm{deg})$
FIG. 3. Final energy and ejection angle distributions of electrons ionized from hydrogenlike $\mathrm{Ar}^{17+}$ ions interacting with a $1 \mathrm{PW}, 800 \mathrm{~nm}$, Gaussianshaped laser beam. The FWHM $\tau$ is 10 laser cycles. The top and bottom rows correspond to beam waists of $1 \mu \mathrm{m}$ and $2 \mu \mathrm{m}$, respectively, while the left and right columns correspond to ions initially located either before $\left(z_{0}<0\right)$ or after $\left(z_{0}>0\right)$ the laser focus. The peak intensities are 6.37 and $1.59 \times 10^{22} \mathrm{~W} / \mathrm{cm}^{2}$ for the beam waists 1 and $2 \mu \mathrm{m}$, respectively. The color scale gives the percentage of ionized electrons. dependence on initial location is found for a beam waist of $2 \mu \mathrm{m}$ [Figs. 3(c) and 3(d)], in which the electrons initially located after the focus are accelerated to higher energy and ejected at smaller angles than those located before the focus.

Comparing Figs. 3(b) and 3(d), we observe that the highest energy gains in Fig. 3(d) are lower than those in Fig. 3(b), which is not surprising since the peak intensity in the former is four times smaller than in the latter. On the other hand, the most probable energy gain $[0.22 \mathrm{GeV}$, indicated by the dark (red) area] in Fig. 3(d) is actually higher than that $(0.15 \mathrm{GeV})$ in Fig. 3(b). The reason for this is the longer Rayleigh length for the laser field in Fig. 3(d), so that electrons can be accelerated for a longer time, thus acquiring more energy. Clearly, the final electron energy gain depends on both the peak intensity and the Rayleigh length of the laser field, both of which are determined by the laser beam waist.

The maximum energy gain of electrons in Fig. 3 is only about $0.4 \mathrm{GeV}$, despite peak intensities of the order of $10^{22} \mathrm{~W} / \mathrm{cm}^{2}$. More intense laser fields are thus needed to increase the energy gain of the accelerated electrons to $1 \mathrm{GeV}$. Simulation results for $\mathrm{Ar}^{17+}$ ions in a $5 \mathrm{PW}$ laser beam focused to 4 and $5 \mu \mathrm{m}$ are shown in Fig. 4. The advantages of placing the ion targets after the focus rather than before the focus are even more clearly visible.

Comparing Figs. 3 and 4, we draw the following conclusions:

(1) Both the final energies and the ejection angles of the accelerated electrons depend on the initial positions of the parent ions, with those located after the focus preferred for higher energy gains and smaller ejection angles.

(2) The maximum energy gain of the accelerated electrons for the 5 PW laser in Fig. 4 approaches $0.8 \mathrm{GeV}$, which

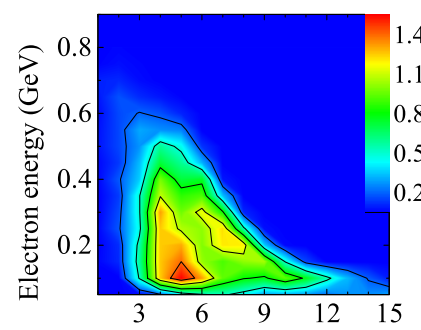

(a)

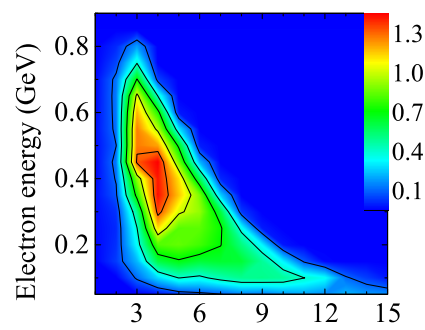

(b) Ejection angle $\theta(\mathrm{deg})$

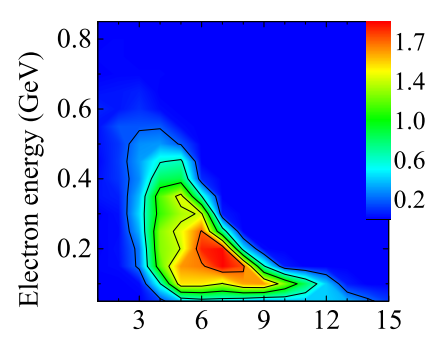

(c)

Ejection angle $\theta(\mathrm{deg})$

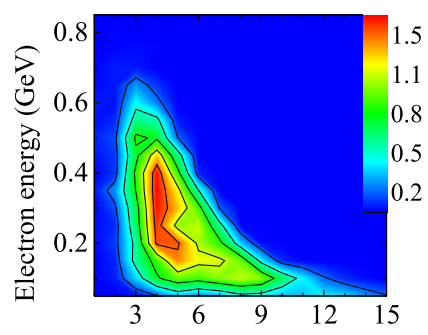

(d)

Ejection angle $\theta(\mathrm{deg})$

FIG. 4. Final energy and ejection angle distributions for electrons ionized from hydrogen-like $\mathrm{Ar}^{17+}$ ions interacting with a $5 \mathrm{PW}$ laser beam having the same parameters as in Fig. 3 but focused to different beam waists. The peak intensities are 2 and $1.27 \times 10^{22} \mathrm{~W} / \mathrm{cm}^{2}$ for beam waists of $4 \mu \mathrm{m}$ and $5 \mu \mathrm{m}$, respectively. 
is twice that of the maximum energy for the $1 \mathrm{PW}$ laser in Fig. 3, even through the peak intensity in Fig. 3 is higher than that in Fig. 4. This may be explained by the different beam waists used: a larger beam waist means a longer Rayleigh length, so that electrons can be accelerated for a longer time, thus acquiring more energy.

(3) Comparing the energy and angle distributions for different beam waists [Figs. 4(a) and 4(b) versus Figs. 4(c) and 4(d)], we find that the initial position dependence is less significant for the greater beam waist, i.e., for a smaller diffraction parameter $s$.

Figure 5 shows the final energy and ejection angle distributions for electrons ionized from $\mathrm{Ar}^{17+}$ ions interacting with a 25 PW laser beam. In Fig. 5(b), one sees that the most probable energy [i.e., the dark (red) area] of the accelerated electrons reaches $1 \mathrm{GeV}$, and the highest energy gain even approaches $2 \mathrm{GeV}$. The three conclusions above summarizing the results in Figs. 3 and 4 are valid also for the results in Fig. 5. In brief, the highest energies and smallest scattering angles for the accelerated electrons are obtained by locating the target ions after the focus.

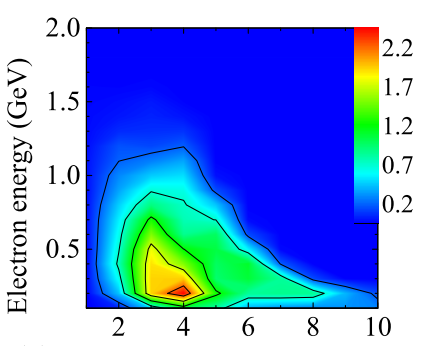

(a)

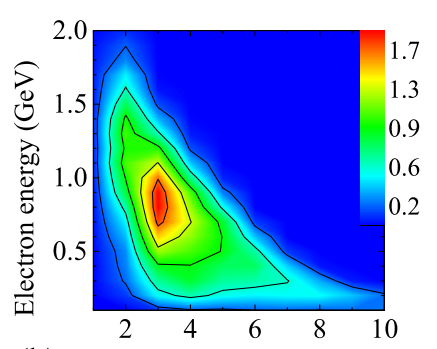

(b)
Ejection angle $\theta$ (deg)

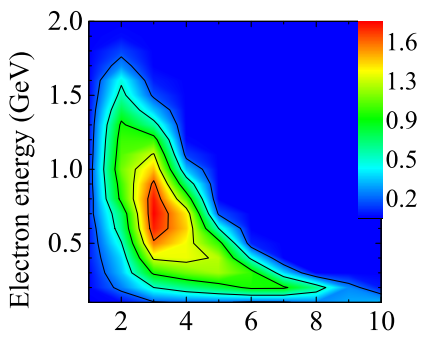

(d)

Ejection angle $\theta(\mathrm{deg})$

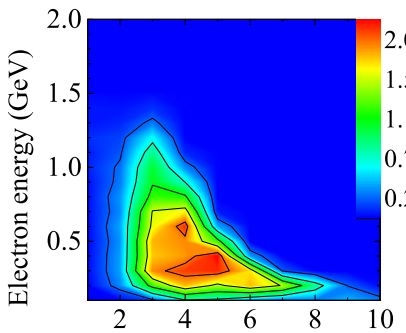

(c)

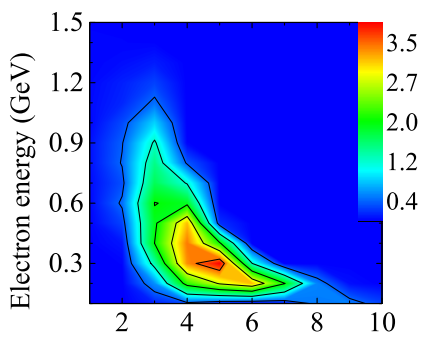

(e)

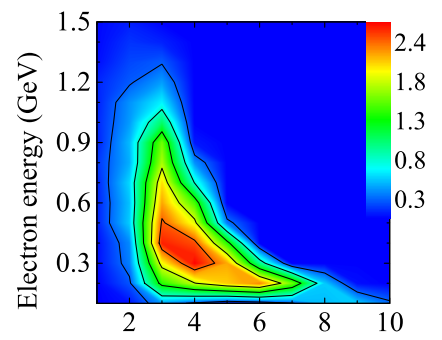

(f)

Ejection angle $\theta(\mathrm{deg})$

FIG. 5. Final energy and ejection angle distributions for electrons ionized from hydrogen-like $\mathrm{Ar}^{17+}$ ions interacting with a $25 \mathrm{PW}$ laser beam having the same parameters as in Fig. 3 but focused to different beam waists. The peak intensities are $6.37,3.25$, and $1.97 \times 10^{22} \mathrm{~W} / \mathrm{cm}^{2}$ for beam waists of 5 , 7 , and $9 \mu \mathrm{m}$, respectively.

\section{Longitudinal components of the electromagnetic field}

For an electron born at rest in a plane wave laser field, the energy absorbed from the field as a function of ejection angle can be simply described by the equation

$$
\varepsilon=\frac{2 m c^{2}}{\tan ^{2} \theta},
$$

which is derived in Ref. 73 from the relativistic momentum and energy conservation law. Laser acceleration of electrons located in highly-charged ions by a paraxial Gaussian beam can also be characterized by this equation. ${ }^{38,44}$ For a tightlyfocused laser beam, however, non-paraxial effects must be taken into account, i.e., the longitudinal components [cf. Eqs. (17c) and (17d)] of the field will suppress the overall energy gain of the electrons, and also replace the monotonic energy-angle relationship by a broadened distribution. ${ }^{44}$

In contrast to the tightly-focused, intense laser beam results shown in Figs. 3-5, in Fig. 6, we present final energy and ejection angle distributions for electrons ionized from hydrogen-like $\mathrm{Ne}^{9+}$ ions interacting with a 1 PW looselyfocused laser beam. In this case, the energy and angle distributions are narrow bands lying along the curve defined by Eq. (20), and are not significantly different for target ions initially located either before or after the laser focus. The reason is that the results in Fig. 6 are for a laser beam with lower peak intensity and larger beam waist (i.e., smaller $s$ ), thus leading to weaker longitudinal electromagnetic fields in Eqs. (17c) and (17d). Thus, the energy and angle distributions look more like those for a paraxial laser field.

The dependence of the ionized electron energy and angular distributions on the positions of the target ions relative to

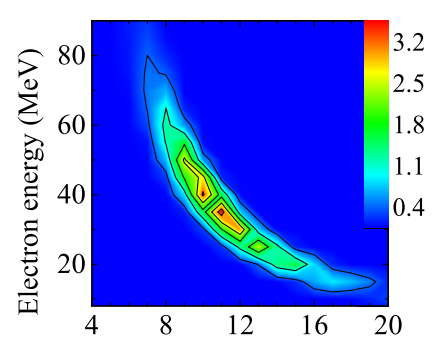

(a)

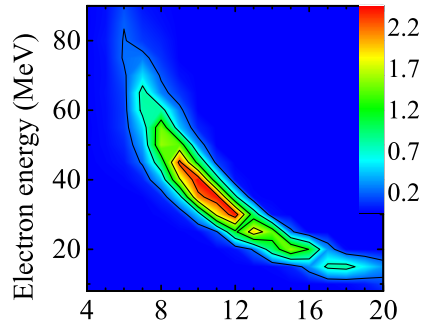

(b)
Ejection angle $\theta(\mathrm{deg})$

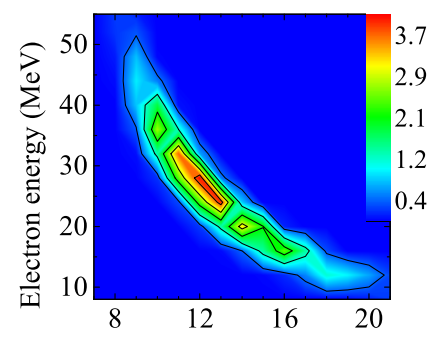

(c) Ejection angle $\theta(\mathrm{deg})$

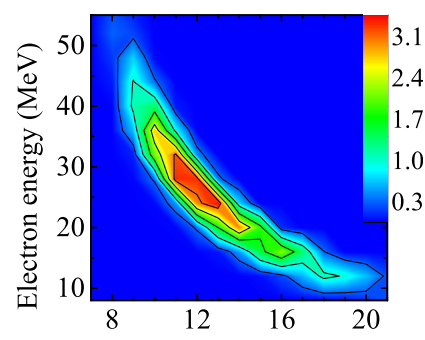

(d)

Ejection angle $\theta(\mathrm{deg})$

FIG. 6. Final energy and ejection angle distributions for electrons ionized from hydrogen-like $\mathrm{Ne}^{9+}$ ions interacting with a $1 \mathrm{PW}$ laser beam having the same parameters as in Fig. 3 but focused to different beam waists. The peak intensities are 1.3 and $0.64 \times 10^{21} \mathrm{~W} / \mathrm{cm}^{2}$ for beam waists of 7 and $10 \mu \mathrm{m}$, respectively. 
the laser focus is a consequence of the longitudinal components of the laser fields in the focal region. Plots of the real part of the spatial profiles of $E_{x}^{(0)}$ and $E_{z}^{(1)}$ fields in the $x z$ plane centered on the laser focus at the origin are presented in Fig. 7. One sees that $E_{x}^{(0)}$, the zero-order component of the laser electric field, is symmetric with respect to the laser focus and has a uniform direction. On the other hand, the direction of the lowest order longitudinal field component, $E_{z}^{(1)}$, flips its sign from quadrant to quadrant in the $x z$-plane. For sufficiently strong laser magnetic field components, it is well known that a charged particle in the field will drift along the propagation direction of the laser beam. The drift velocity $\mathbf{v}_{d r}$ is proportional to $\mathbf{E} \times \mathbf{B}$. Under the influence of the magnetic field $B_{y}^{(0)}$, the electric fields plotted in Fig. 7 will give a dominant drift along the $+z$ direction, $v_{z}^{(0)} \sim E_{x}^{(0)} B_{y}^{(0)}$, and a minor drift along the $x$ direction, $v_{x}^{(1)} \sim-E_{z}^{(1)} B_{y}^{(0)}$, as shown in Fig. 8. The direction of $v_{x}^{(1)}$ depends on the direction of $E_{z}^{(1)}$ in different quadrants. Also, $v_{x}^{(1)}$ vanishes along both the $x$-axis and the $z$-axis. Most important, one sees that $v_{x}^{(1)}$ points
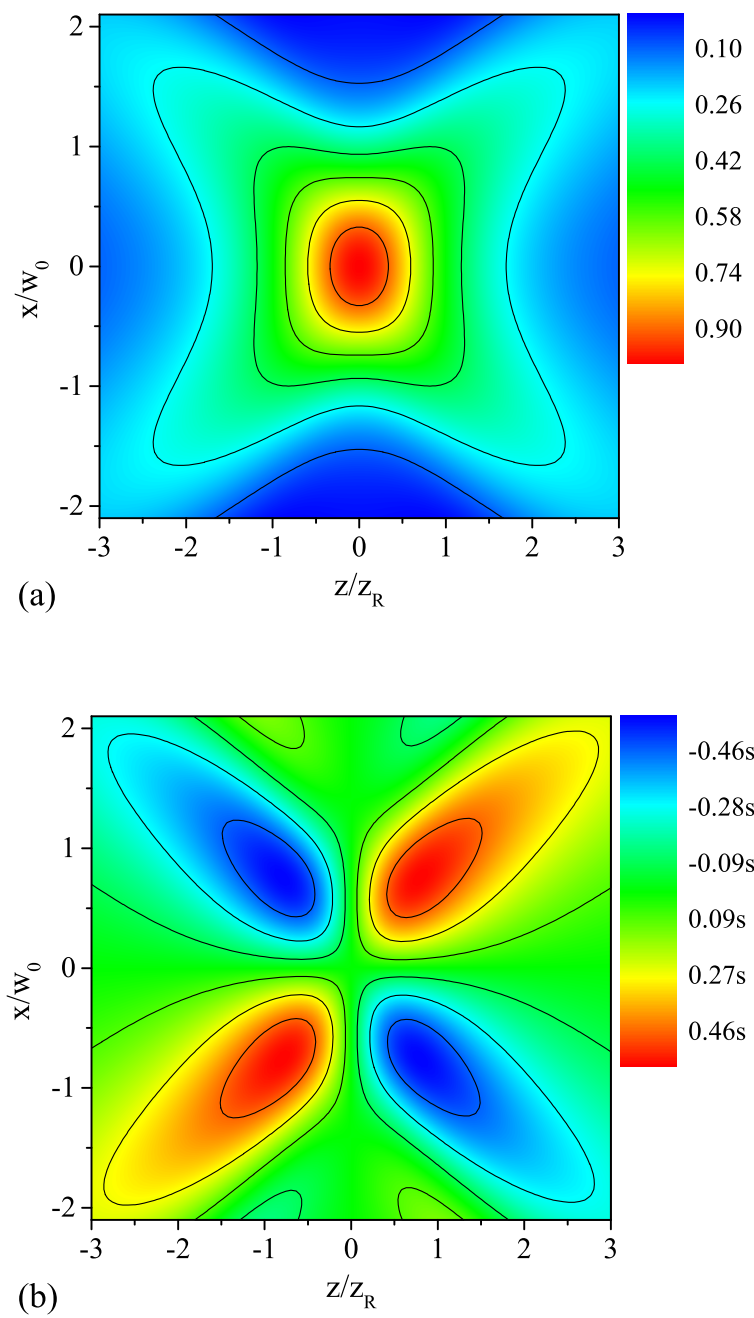

FIG. 7. The real part of the spatial profiles of the laser electric field components $E_{x}^{(0)}$ and $E_{z}^{(1)}$ in the $x z$-plane: (a) spatial profile of the zero-order field $\operatorname{Re}\left(\psi_{0}\right)$ [cf. Eq. (17a)], and (b) spatial profile of the 1st-order longitudinal field $\operatorname{Re}\left(-2 s Q \xi \psi_{0}\right)$ [cf. Eq. (17c)]. The longitudinal field $E_{z}^{(1)}$ is smaller than the main component $E_{x}^{(0)}$ by one order of $s$. The color scales give the relative amplitudes and directions of each field.
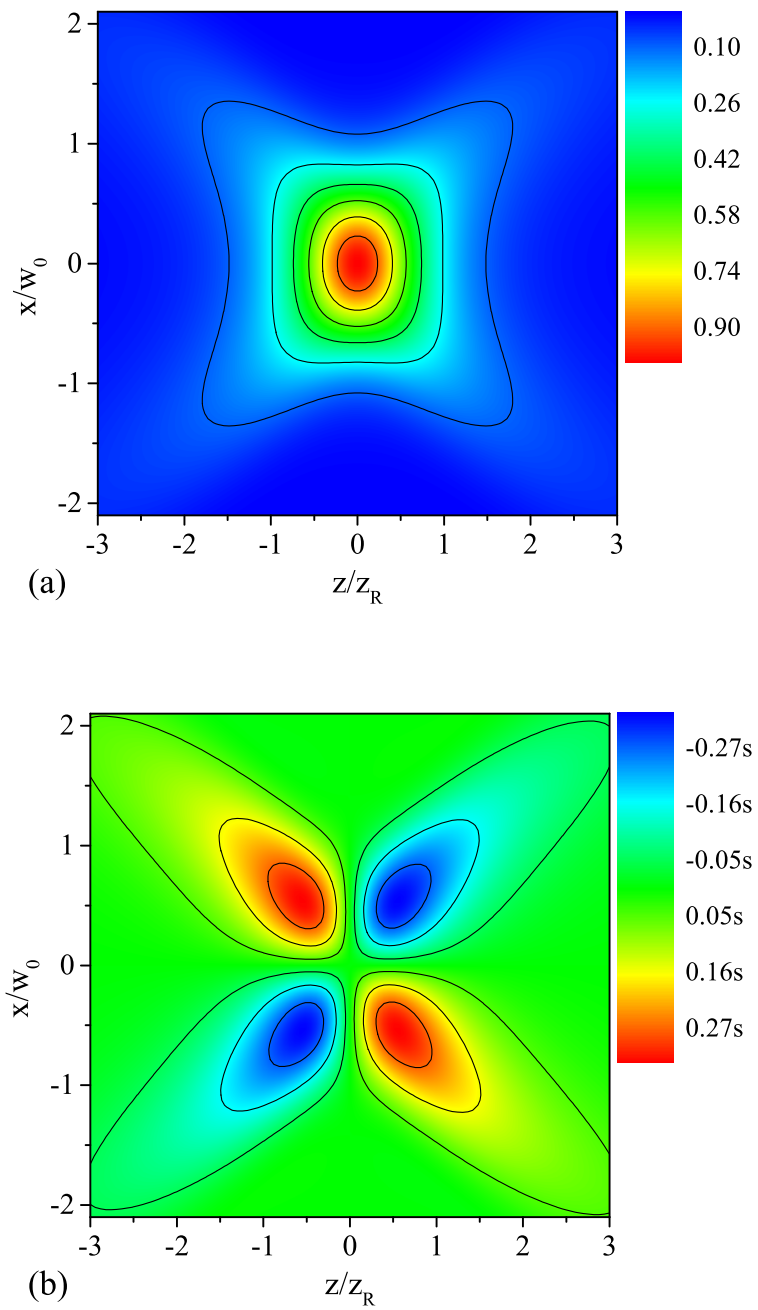

FIG. 8. The spatial profiles of the drift velocities in the $x z$-plane: (a) along the propagation direction, $v_{z}^{(0)} \sim E_{x}^{(0)} B_{y}^{(0)} \sim\left[\operatorname{Re}\left(\psi_{0}\right)\right]^{2}$, and (b) along the polarization direction, $v_{x}^{(1)} \sim-E_{z}^{(1)} B_{y}^{(0)} \sim \operatorname{Re}\left(2 s Q \xi \psi_{0}\right) \operatorname{Re}\left(\psi_{0}\right) . v_{x}^{(1)}$ is smaller than $v_{z}^{(0)}$ by one order of $s$. The color scales give the relative amplitudes and directions of each drift velocity.

towards the $z$-axis for $z>0$, and points away from the $z$-axis for $z<0$. The direction of $v_{x}^{(1)}$ implies that electrons ionized before the focus will be pushed away from the laser focus, while those ionized after the laser focus will be pushed toward the $z$-axis. This explains qualitatively why electrons ionized before the focus are scattered to lower energies and larger angles, while electrons ionized in the region beyond the focus will drift towards the propagation axis, and hence experience stronger intensity, thus leading to greater energy gains and smaller ejection angles.

\section{CONCLUSIONS}

In this paper, relativistic classical trajectory Monte Carlo methods have been used to simulate electron ionization and acceleration from hydrogen-like highly-charged ions interacting with an intense, tightly-focused laser field. A relativistic microcanonical ensemble is prepared corresponding to the ground states of both $\mathrm{Ar}^{17+}$ and $\mathrm{Ne}^{9+}$ hydrogenlike ions, and non-paraxial Gaussian beams are employed for an accurate description of a tightly-focused laser beam, 
including corrections up to fifth-order in the diffraction parameter $s$. Numerical simulations have been carried out for petawatt lasers currently or soon-to-be available. It is found that the final energy and ejection angle distributions of the ionized electrons depend on the initial positions of their parent ions relative to the laser focus. The electrons initially located after the focus generally acquire more energy and are ejected to smaller angles. The energy and angle dependence on the initial target ion positions decreases as the diffraction parameter $s$ becomes smaller. In contrast, the authors of Refs. 51 and 52 found that the electrons initially located before the focus acquire more energy. These seemingly contradictory conclusions originate from the fact that the laser fields investigated in Refs. 51 and 52 are tightly-focused by a parabolic mirror and are different from the laser fields employed in this paper. As shown in Fig. 11 of Ref. 51, the longitudinal electric field is responsible for the acceleration of electrons and is several times larger than the transverse electric field in their simulations. On the other hand, as has been shown in Fig. 7 above, the transverse electric field is the dominant field component in our simulations.

We also find that the peak intensity of the laser field is not the only parameter that affects the energy gain of the accelerated electrons. The Rayleigh length also plays an important role: the longer the Rayleigh length, the longer the time the electrons experience the intense laser field, and thus, the greater the energy gain. Moreover, examination of the spatial distribution of the longitudinal electric field $E_{z}^{(1)}$ (i.e., the lowest-order non-paraxial field) reveals an asymmetric drift along the polarization direction, which explains qualitatively the dependence of the final energy and angle distributions on the initial positions of the target ions from which the electrons are ionized.

Note, finally, that relativistic classical trajectory Monte Carlo methods are not able to take into account either multiphoton or tunneling ionization processes, which are quantum effects. Ionization in our calculations occurs by over-the-barrier ionization. Nevertheless, the precise ionization mechanism does not change the physics of laser acceleration treated in this work. By whatever mechanism electrons are placed in the continuum in the presence of an intense, tightly-focused laser field, their subsequent trajectories and final energy and angular distributions will be as presented here. The main consequence for experiments will be that ionization by multiphoton and tunneling processes will occur at lower intensities than for over-the-barrier ionization. This should thus be taken into account in planning experiments. Specifically, one must choose hydrogen-like ions such that when ionization is most likely, the laser field is at its peak intensity. We expect that the findings presented here will serve as a useful guide for experiments aimed at optimizing the energy gain resulting from laser acceleration of electrons.

\section{ACKNOWLEDGMENTS}

We thankfully acknowledge helpful conversations with Shouyuan Chen and Donald P. Umstadter concerning experimental aspects of petawatt laser interactions with atoms and plasmas. This research was supported in part by the Department of Energy, Office of Science, Division of Chemical Sciences, Geosciences, and Biosciences, under Grant No. DE-FG03-96ER14646 at the University of Nebraska-Lincoln, and by the National Science Foundation under Grant No. NSF PHY11-25915 at the Kavli Institute for Theoretical Physics, University of California at Santa Barbara. Our simulations were completed utilizing the Holland Computing Center of the University of NebraskaLincoln. L.-W. Pi and A. F. Starace gratefully acknowledge the hospitality of the Kavli Institute for Theoretical Physics, where part of this article was prepared. S. X. Hu acknowledges the research support of the DOE National Nuclear Security Administration under Award No. DENA0001944, the University of Rochester, and the New York State Energy Research and Development Authority.

${ }^{1}$ A. Di Piazza, C. Müller, K. Z. Hatsagortsyan, and C. H. Keitel, Rev. Mod. Phys. 84, 1177 (2012).

${ }^{2}$ C. Liu, S. Banerjee, J. Zhang, S. Chen, K. Brown, J. Mills, N. Powers, B. Zhao, G. Golovin, I. Ghebregziabher, and D. Umstadter, Proc. SPIE 8599, 859919 (2013).

${ }^{3}$ D. Umstadter, J. Phys. D: Appl. Phys. 36, R151 (2003).

${ }^{4}$ W. Leemans and E. Esarey, Phys. Today 62(3), 44 (2009).

${ }^{5}$ E. Esarey, C. B. Schroeder, and W. P. Leemans, Rev. Mod. Phys. 81, 1229 (2009).

${ }^{6}$ H.-P. Schlenvoigt, O. Jackel, S. M. Pfotenhauer, and M. C. Kaluza, in Advances in Solid-State Lasers: Development and Applications, edited by M. Grishin (InTech, 2010), Chap. 24.

${ }^{7}$ S. Y. Kalmykov, A. Beck, X. Davoine, E. Lefebvre, and B. A. Shadwick, New J. Phys. 14, 033025 (2012).

${ }^{8}$ X. Wang et al., Nat. Commun. 4, 1988 (2013).

${ }^{9}$ H. T. Kim, K. H. Pae, H. J. Cha, I. J. Kim, T. J. Yu, J. H. Sung, S. K. Lee, T. M. Jeong, and J. Lee, Phys. Rev. Lett. 111, 165002 (2013).

${ }^{10}$ W. P. Leemans, A. J. Gonsalves, H.-S. Mao, K. Nakamura, C. Benedetti, C. B. Schroeder, Cs. Tóth, J. Daniels, D. E. Mittelberger, S. S. Bulanov, J.-L. Vay, C. G. R. Geddes, and E. Esarey, Phys. Rev. Lett. 113, 245002 (2014).

${ }^{11}$ A. J. Gonsalves, K. Nakamura, J. Daniels, H.-S. Mao, C. Benedetti, C. B. Schroeder, Cs. Tóth, J. van Tilborg, D. E. Mittelberger, S. S. Bulanov, J.L. Vay, C. G. R. Geddes, E. Esarey, and W. P. Leemans, Phys. Plasmas 22, 056703 (2015).

${ }^{12}$ B. Quesnel and P. Mora, Phys. Rev. E 58, 3719 (1998).

${ }^{13}$ F. V. Hartemann, J. R. Van Meter, A. L. Troha, E. C. Landahl, N. C. Luhmann, H. A. Baldis, A. Gupta, and A. K. Kerman, Phys. Rev. E 58, 5001 (1998)

${ }^{14}$ Y. I. Salamin and C. H. Keitel, Phys. Rev. Lett. 88, 095005 (2002).

${ }^{15}$ Y. I. Salamin, G. R. Mocken, and C. H. Keitel, Phys. Rev. Spec. Top. Accel. Beams 5, 101301 (2002).

${ }^{16}$ J. Pang, Y. K. Ho, X. Q. Yuan, N. Cao, Q. Kong, P. X. Wang, L. Shao, E. H. Esarey, and A. M. Sessler, Phys. Rev. E 66, 066501 (2002).

${ }^{17}$ D. Cline, L. Shao, X. Ding, Y. Ho, Q. Kong, and P. Wang, J. Mod. Phys. 04, 1 (2013).

${ }^{18}$ M. Thévenet, A. Leblanc, S. Kahaly, H. Vincenti, A. Vernier, F. Quéré, and J. Faure, "Vacuum laser acceleration of relativistic electrons using plasma mirror injectors," Nature Phys. (in review).

${ }^{19}$ Y. I. Salamin, Phys. Rev. A 73, 043402 (2006).

${ }^{20}$ D. N. Gupta, N. Kant, D. E. Kim, and H. Suk, Phys. Lett. A 368, 402 (2007).

${ }^{21}$ L. Dai, J.-X. Li, W.-P. Zang, and J.-G. Tian, Opt. Express 19, 9303 (2011).

${ }^{22}$ S. Payeur, S. Fourmaux, B. E. Schmidt, J. P. MacLean, C. Tchervenkov, F. Légaré, M. Piché, and J. C. Kieffer, Appl. Phys. Lett. 101, 041105 (2012).

${ }^{23}$ C. Varin, S. Payeur, V. Marceau, S. Fourmaux, A. April, B. Schmidt, P.-L. Fortin, N. Thiré, T. Brabec, F. Légaré, J.-C. Kieffer, and M. Piché, Appl. Sci. 3, 70 (2013).

${ }^{24}$ V. Marceau, C. Varin, T. Brabec, and M. Piché, Phys. Rev. Lett. 111, 224801 (2013).

${ }^{25}$ V. Marceau, P. Hogan-Lamarre, T. Brabec, M. Piché, and C. Varin, J. Phys. B: At. Mol. Opt. Phys. 48, 045601 (2015). 
${ }^{26}$ K. P. Singh, Appl. Phys. Lett. 87, 254102 (2005).

${ }^{27}$ F. Sohbatzadeh, S. Mirzanejhad, and M. Ghasemi, Phys. Plasmas 13, 123108 (2006).

${ }^{28}$ F. Sohbatzadeh, S. Mirzanejhad, and H. Aku, Phys. Plasmas 16, 023106 (2009).

${ }^{29}$ D. N. Gupta, H. J. Jang, and H. Suk, J. Appl. Phys. 105, 106110 (2009).

${ }^{30}$ J.-X. Li, W.-P. Zang, and J.-G. Tian, Appl. Phys. Lett. 96, 031103 (2010).

${ }^{31}$ X. Y. Wu, P. X. Wang, and S. Kawata, Appl. Phys. Lett. 100, 221109 (2012).

${ }^{32}$ Y. I. Salamin, Phys. Lett. A 376, 2442 (2012).

${ }^{33}$ Y. I. Salamin and N. M. Jisrawi, J. Phys. B: At. Mol. Opt. Phys. 47, 025601 (2014).

${ }^{34}$ H. Liu, X. T. He, and S. G. Chen, Phys. Rev. E 69, 066409 (2004).

${ }^{35}$ K. P. Singh, J. Appl. Phys. 100, 044907 (2006).

${ }^{36}$ H. S. Ghotra and N. Kant, Appl. Phys. B 120, 141 (2015).

${ }^{37}$ M. Vaziri, M. Golshani, S. Sohaily, and A. Bahrampour, Phys. Plasmas 22, 033118 (2015).

${ }^{38}$ S. X. Hu and A. F. Starace, Phys. Rev. Lett. 88, 245003 (2002).

${ }^{39}$ S. X. Hu and A. F. Starace, Phys. Rev. E 73, 066502 (2006).

${ }^{40}$ M. Lax, W. H. Louisell, and W. B. McKnight, Phys. Rev. A 11, 1365 (1975).

${ }^{41}$ G. P. Agrawal and D. N. Pattanayak, J. Opt. Soc. Am. 69, 575 (1979).

${ }^{42}$ L. W. Davis, Phys. Rev. A 19, 1177 (1979).

${ }^{43}$ J. P. Barton and D. R. Alexander, J. Appl. Phys. 66, 2800 (1989).

${ }^{44}$ A. Maltsev and T. Ditmire, Phys. Rev. Lett. 90, 053002 (2003).

${ }^{45}$ S. Masuda, M. Kando, H. Kotaki, and K. Nakajima, Phys. Plasmas 12, 013102 (2005).

${ }^{46}$ S. M. Sepke and D. P. Umstadter, Opt. Lett. 31, 1447 (2006).

${ }^{47}$ S. M. Sepke and D. P. Umstadter, Opt. Lett. 31, 2589 (2006).

${ }^{48}$ S. M. Sepke and D. P. Umstadter, J. Opt. Soc. Am. B 23, 2295 (2006).

${ }^{49}$ Y. I. Salamin, Appl. Phys. B 86, 319 (2007).

${ }^{50}$ J. Li, W. Zang, and J. Tian, Opt. Express 17, 4959 (2009).

${ }^{51}$ K. I. Popov, V. Yu. Bychenkov, W. Rozmus, and R. D. Sydora, Phys. Plasmas 15, 013108 (2008).
${ }^{52}$ S. G. Bochkarev, K. I. Popov, and V. Y. Bychenkov, Plasma Phys. Rep. 37, 603 (2011).

${ }^{53}$ J. S. Roman, L. Roso, and H. R. Reiss, J. Phys. B: At. Mol. Opt. Phys. 33, 1869 (2000).

${ }^{54}$ H. Bauke and C. H. Keitel, Comput. Phys. Commun. 182, 2454 (2011).

${ }^{55}$ H. Bauke, H. G. Hetzheim, G. R. Mocken, M. Ruf, and C. H. Keitel, Phys. Rev. A 83, 063414 (2011).

${ }^{56}$ V. S. Popov, B. M. Karnakov, and V. D. Mur, JETP Lett. 79, 262 (2004)

${ }^{57}$ M. Klaiber, E. Yakaboylu, and K. Z. Hatsagortsyan, Phys. Rev. A 87, 023418 (2013).

${ }^{58}$ J.-H. Yang, R. S. Craxton, and M. G. Haines, Plasma Phys. Controlled Fusion 53, 125006 (2011).

${ }^{59}$ H. Schmitz, K. Boucke, and H.-J. Kull, Phys. Rev. A 57, 467 (1998).

${ }^{60}$ D. A. Burton and A. Noble, Contemp. Phys. 55, 110 (2014).

${ }^{61}$ I. Ghebregziabher and B. C. Walker, in Advances in Solid-State Lasers: Development and Applications, edited by M. Grishin (InTech, 2010), Chap. 23.

${ }^{62}$ E. E. Fill, J. Opt. Soc. Am. B 11, 2241 (1994).

${ }^{63}$ E. Esarey, P. Sprangle, J. Krall, and A. Ting, IEEE J. Quantum Electron. 33, 1879 (1997).

${ }^{64}$ S. Hooker, in Laser-Plasma Interactions, edited by D. A. Jaroszynski, R. A. Bingham, and R. A. Cairns (CRC Press, Boca Raton, FL, 2009), Chap. 3.

${ }^{65}$ R. Abrines and I. C. Percival, Proc. Phys. Soc. 88, 861 (1966).

${ }^{66}$ R. Abrines and I. C. Percival, Proc. Phys. Soc. 88, 873 (1966).

${ }^{67}$ J. G. Leopold and I. C. Percival, J. Phys. B: At. Mol. Phys. 12, 709 (1979).

${ }^{68}$ L. N. Gaier and C. H. Keitel, Phys. Rev. A 65, 023406 (2002).

${ }^{69}$ H. G. Hetzheim and C. H. Keitel, Phys. Rev. Lett. 102, 083003 (2009).

${ }^{70}$ P. D. Grugan, S. Luo, M. Videtto, C. Mancuso, and B. C. Walker, Phys. Rev. A 85, 053407 (2012).

${ }^{71}$ R. D. Sard, Relativistic Mechanics (Benjamin, New York, 1970), p. 189.

${ }^{72}$ J. T. Zhang, P. X. Wang, Q. Kong, Z. Chen, and Y. K. Ho, Nucl. Instrum. Methods Phys. Res. A 580, 1169 (2007).

${ }^{73}$ C. I. Moore, J. P. Knauer, and D. D. Meyerhofer, Phys. Rev. Lett. 74, 2439 (1995). 